\title{
ODAE: Ontology-based systematic representation and analysis of drug adverse events and its usage in study of adverse events given different patient age and disease conditions
}

Hong Yu ${ }^{1,2^{*}}$, Solomiya Nysak ${ }^{3}$, Noemi Garg ${ }^{4}$, Edison Ong ${ }^{5}$, Xianwei Ye ${ }^{1,2}$, Xiangyan Zhang ${ }^{1,2}$ and Yongqun He ${ }^{6 *}$ From The 12th International Conference on Computational Systems Biology (ISB 2018)

Guiyang, China. 18-21 August 2018

\begin{abstract}
Background: Drug adverse events (AEs), or called adverse drug events (ADEs), are ranked one of the leading causes of mortality. The Ontology of Adverse Events (OAE) has been widely used for adverse event AE representation, standardization, and analysis. OAE-based ADE-specific ontologies, including ODNAE for drug-associated neuropathyinducing AEs and OCVDAE for cardiovascular drug AEs, have also been developed and used. However, these ADEspecific ontologies do not consider the effects of other factors (e.g., age and drug-treated disease) on the outcomes of ADEs. With more ontological studies of ADEs, it is also critical to develop a general purpose ontology for representing ADEs for various types of drugs.

Results: Our survey of FDA drug package insert documents and other resources for 224 neuropathy-inducing drugs discovered that many drugs (e.g., sirolimus and linezolid) cause different AEs given patients' age or the diseases treated by the drugs. To logically represent the complex relations among drug, drug ingredient and mechanism of action, $A E$, age, disease, and other related factors, an ontology design pattern was developed and applied to generate a community-driven open-source Ontology of Drug Adverse Events (ODAE). The ODAE development follows the OBO Foundry ontology development principles (e.g., openness and collaboration). Built on a generalizable ODAE design pattern and extending the OAE and NDF-RT ontology, ODAE has represented various AEs associated with the over 200 neuropathy-inducing drugs given different age and disease conditions. ODAE is now deposited in the Ontobee for browsing and queries. As a demonstration of usage, a SPARQL query of the ODAE knowledge base was developed to identify all the drugs having the mechanisms of ion channel interactions, the diseases treated with the drugs, and AEs after the treatment in adult patients. AE-specific drug class effects were also explored using ODAE and SPARQL.

(Continued on next page)
\end{abstract}

\footnotetext{
* Correspondence: yuhong20040416@sina.com; yongqunh@med.umich.edu 'Department of Pulmonary and Critical Care Medicine, Guizhou Provincial People's Hospital, Guiyang 550002, Guizhou, China

${ }^{6}$ Unit for Laboratory Animal Medicine, Department of Microbiology and Immunology, Center for Computational Medicine and Bioinformatics, and Comprehensive Cancer Center, University of Michigan Medical School, Ann Arbor, Ml 48109, USA

Full list of author information is available at the end of the article
}

(c) The Author(s). 2019 Open Access This article is distributed under the terms of the Creative Commons Attribution 4.0 International License (http://creativecommons.org/licenses/by/4.0/), which permits unrestricted use, distribution, and reproduction in any medium, provided you give appropriate credit to the original author(s) and the source, provide a link to the Creative Commons license, and indicate if changes were made. The Creative Commons Public Domain Dedication waiver (http://creativecommons.org/publicdomain/zero/1.0/) applies to the data made available in this article, unless otherwise stated. 
(Continued from previous page)

Conclusion: ODAE provides a general representation of ADEs given different conditions and can be used for querying scientific questions. ODAE is also a robust knowledge base and platform for semantic and logic representation and study of ADEs of more drugs in the future.

Keywords: Drug, Ontology, Drug adverse event, Ontology of drug adverse events, ODAE, Bioinformatics

\section{Background}

As defined by the US FDA, an adverse drug event (ADE), also call adverse drug reaction is any untoward medical occurrence associated with the use of a drug in humans, whether or not considered drug related. ADEs are far more than one would think. ADEs are ranked as the fourth leading cause of death in the United States and Canada behind heart disease, cancer, and stroke [1]. In Europe, approximately 5\% of all hospital admissions are caused by ADEs, and ADEs cause 197,000 deaths annually throughout the EU [2]. ADEs are estimated as the sixth leading cause of death worldwide. ADE-related death rates are associated with age, race, and urbanization subgroups [3]. However, how ADEs are associated with different factors is still unclear and not well studied.

Ontologies play critical roles in data science to facilitate data normalization, integration, processing, and analyses [4-7]. Biological/biomedical ontologies are sets of computer- and human-interpretable terms and relations that represent entities in the biological/biomedical world and how they relate to each other [8]. Ontologies have been widely used in various areas. For example, the NCBI Taxonomy ontology (NCBITaxon) includes the names and hierarchy of over one million of taxonomy names [9]. The Biological Pathway Exchange (BioPAX) standard ontology targets for representing molecular and cellular pathways and facilitates the exchange of biological pathway data [10]. The Gene Ontology (GO) [11] has been widely used to support gene expression data analyses, literature mining, and knowledge representation. Since its publication in 2000, the original GO paper [11] has been cited by over 20,000 publications. The Ontology for Biomedical Investigations (OBI) [12], co-developed by over 20 biomedical communities, provides integrative representations of data in various areas of life-science and clinical investigations [13-16]. Ontologies are also being used to support various metadata generations $[15,17,18]$, leading to reproducible research, and data FAIRness (findable, accessible, interoperable, and reusable) [5, 19-21].

The Ontology of Adverse Events (OAE) is a community-based biomedical ontology in the area of adverse events [22, 23]. Under OAE, an adverse event is defined as a pathological bodily process that occurs after the administration of a pharmaceutical product. Such $\mathrm{AE}$ may result in any unfavorable and unintended sign (including an abnormal laboratory finding), symptom, or disease temporally associated with the use of a medicinal product. Aligned with the US FDA definition, an OAE-defined AE does not necessarily have a causal relationship with this treatment. OAE has been used in different applications, such as data integration for nanomaterial risk assessment [24], $\mathrm{AE}$ case report analysis [23], time information representation of AEs for temporal analysis [25], extraction of potential drug AEs from medical case reports [26], and drug class effect analysis [27]. OAE has also been extended to generate the Ontology of Vaccine Adverse Events (OVAE) [28], Ontology of Drug Neuropathy Adverse events (ODNAE) [29], and Ontology of Cardiovascular Drug AEs (OCVDAE) [27]. ODNAE focuses on representing drug-associated neuropathy AEs [29]. OCVDAE focuses on AEs associated with cardiovascular drugs used to treat cardiovascular diseases [27].

There is a critical need to develop a general purpose ontology of drug adverse events. ODNAE and OCVDAE focus on specific areas of ADE domains, cover overlapping drugs, and do not consider the effects of specific conditions such as patient age and drug-treated diseases on the outcomes of ADEs. Most ADE websites only list general ADEs and do not differentiate different AEs under specific age range such as adult, pediatric, and senior ages. Often a drug is used to treat different diseases. Given different diseases treated, the AEs induced by the drug may vary a lot. Although we were developers of the ODNAE and OCVDAE, we realize that it would be very beneficial to have a more general ADE ontology design to cover all kinds of scenarios. Towards this goal, here we report our design, implementation, and application of an open source and community-driven Ontology of Drug Adverse Events (ODAE). ODAE ontologically represents drugs, their chemical ingredients, AEs, patient age, drug treated diseases, and how these entities are related. Our use case is the collection of AEs associated over 200 neuropathy-inducing drugs through manual curation of related FDA package insert documents. We have also demonstrated the usages of ODAE by SPARQL querying for scientific questions and analyzing AE-specific drug class effects. Our studies show that a systematical analysis of the ODAE knowledge allows the identification of scientific insights about these drug AEs given specific age and disease conditions. 


\section{Methods}

\section{Collection of new AEs associated with FDA-approved} neuropathy inducing drugs

While the ODNAE ontology [29] has stored the information of over 200 neuropathy-inducing drugs and their associated neuropathy AEs, ODNAE does not include other non-neuropathy AEs associated with these drugs. Our first effort was to survey FDA package insert documents and other related resources databases such as Drugs@FDA to identify all known AEs associated with these drugs. All data were compiled in an Excel file with a predefined format. Standard ontologies were used to represent related entities. Specifically, all AEs, diseases, and drugs were mapped to specific terms in OAE, the Disease Ontology (DOID) [30], and National Drug File Reference Terminology (NDF-RT) [31], respectively. Some adverse events were not associated with any OAE term, and were therefore compiled in a separate Excel file, which would be later manually annotated and used to update the OAE ontology.

\section{ODAE ontology generation}

The ODAE ontology design follows the Open Biomedical Ontologies (OBO) Foundry principles (e.g., openness and collaboration) [32]. If ever possible, ODAE reuses terms from existing reliable ontologies, for example, NCBITaxon for organisms [9], UBERON for animal anatomical entities [33], the Disease Ontology (DOID) for diseases [30], and NRF-RT for drugs and drug-related information [31], and OAE for AEs [34]. For those AE terms not found in OAE, we added new OAE terms based on the standard OAE development procedure [34]. Ontofox (http://ontofox. hegroup.org/) [8] was used to extract subsets of related terms from different ontologies. Ontorat (http:// ontorat.hegroup.org/) [35] was used to quickly add the annotations and relations between different entities. ODAE is formatted using the W3C standard Web Ontology Language (OWL2) (http://www.w3.org/ TR/owl-guide/). The Protégé 5.0 OWL ontology editor (http://protege.stanford.edu/) was used for manual ontology editing.

\section{ODAE access, visualization, and licensing}

The ODAE project website is located at GitHub: https:// github.com/ODAE-ontology/ODAE. ODAE was also deposited in Ontobee, the default OBO Foundry ontology linked server [36]. The ODAE website in Ontobee is: http://www.ontobee.org/ontology/ODAE. The ODAE source code is freely available under the Creative Commons 3.0 License (http://creativecommons.org/licenses/ by/3.0/), which allows ODAE users to freely distribute and use ODAE.

\section{ODAE ontology knowledge query and analysis}

The ODAE ontology is formatted using the Web Ontology Language (OWL) [37] format. After ODAE is deposited to the Ontobee RDF triple store [36], SPARQL (a recursive acronym for SPARQL Protocol and RDF Query Language) [38] was used to query ODAE to address specific questions using the Ontobee SPARQL query web page (http://www.ontobee.org/sparql). The knowledge stored in the ODAE ontology was also analyzed using tools available under the Protégé OWL editor.

\section{Results}

Collection and analysis of various ADEs associated with 224 neuropathy-inducing drugs

In total 224 neuropathy-inducing drugs were annotated and analyzed in our study. In total 185 AEs were identified amongst these drugs. Only 14 AEs of the 185 AEs were not found in OAE, which were then annotated and added to OAE using standard OAE development protocol. Table 1 lists the top ten AEs associated with these drugs. Nausea is the most commonly identified ADE that occurs in 259 cases. Since each drug may be studied in over one scenario based on age and disease to be treated, the nausea AE cases is more than the total number (i.e., 224) of drugs studied. Other common adverse events include diarrhea, headache, vomiting, dizziness, rash, etc. (Table 1). The study also found that amongst the diseases and disorders treated or prevented by neuropathy inducing drugs are cancer, infections such as bacterial or fungal infections and immune disorders such as acquired immunodeficiency syndrome. Among the 224 neuropathy-inducing drugs we collected, over 30 drugs (e.g., sirolimus, linezolid, and levofloxacin) have been used to treat various respiratory diseases.

Table 1 Top ten ADEs associated with neuropathy-inducing drugs

\begin{tabular}{ll}
\hline Adverse Events & Frequency \\
\hline Nausea & 259 \\
Diarrhea & 173 \\
Headache & 168 \\
Vomiting & 149 \\
Dizziness & 106 \\
Rash & 99 \\
Abdominal Pain & 91 \\
Fatigue & 78 \\
Infection & 75 \\
Constipation & 66 \\
\hline
\end{tabular}




\section{Collection and analysis of the ADE data given different conditions}

Our study found that patient age and disease conditions have significant influence on the final ADE outcomes. For example, sirolimus, also known as rapamycin, is a macrolide compound used to prevent organ transplant rejection and to treat lymphangioleiomyomatosis [39, 40]. Sirolimus has immunosuppressant functions in humans and is commonly useful for preventing the rejection of kidney transplants. Sirolimus inhibits activation of $\mathrm{T}$ cells and $\mathrm{B}$ cells by reducing their sensitivity to interleukin-2 through mTOR inhibition. Sirolimus is also used to treat lymphangioleiomyomatosis, a rare, progressive lung disease that primarily affects women of childbearing age. The common sirolimus AEs associated with its usage for these two treatments include abdominal pain, diarrhea, headache, hypercholesterolemia, nausea, and peripheral edema (Fig. 1a). However, sirolimus also shows many AEs present only during its treatment of lymphangioleiomyomatosis or during its usage in renal transplantation (Fig. 1a). Linezolid is an antibiotic used to treat infections caused by aerobic Gram-positive bacteria (e.g., streptococci and vancomycin-resistant enterococci) [41]. Linezolid is often used for infections of the skin and pneumonia that are resistant to other antibiotics. Linezolid can be used in adults as well as pediatric patients. Our annotation of FDA package insert documents also found shared and differentiated AEs when linezolid is used in these two groups of patients (Fig. 1b).

Our study also found that the age and disease conditions have been found to be an important factor of the AEs of many other drugs, including Ribavirin, Pramipexole (mirapex), Nevirapine, recombinant Interferon-2a (Roferon A), Infliximab (remicade), Capecitabine (xeloda), and Alosetron (lotronex). As a drug example, Infliximab (remicade) is associated with different AEs given different ages (e.g., adults and pediatric), and disease conditions (i.e., Crohn's disease, and ulcerative colitis). For instance, our manual annotation found that Infliximab is statistically significantly associated with neutropenia and leukopenia in Crohn's disease patients but not in ulcerative colitis patients, and associated with pharyngitis and abdominal pain in ulcerative colitis patients but not in Crohn's disease patients.

\section{ODAE design and development}

Figure 2 shows the basic top-level hierarchical structure of ODAE. Specifically, ODAE uses and is aligned with the Basic Formal Ontology (BFO) [42] upper level

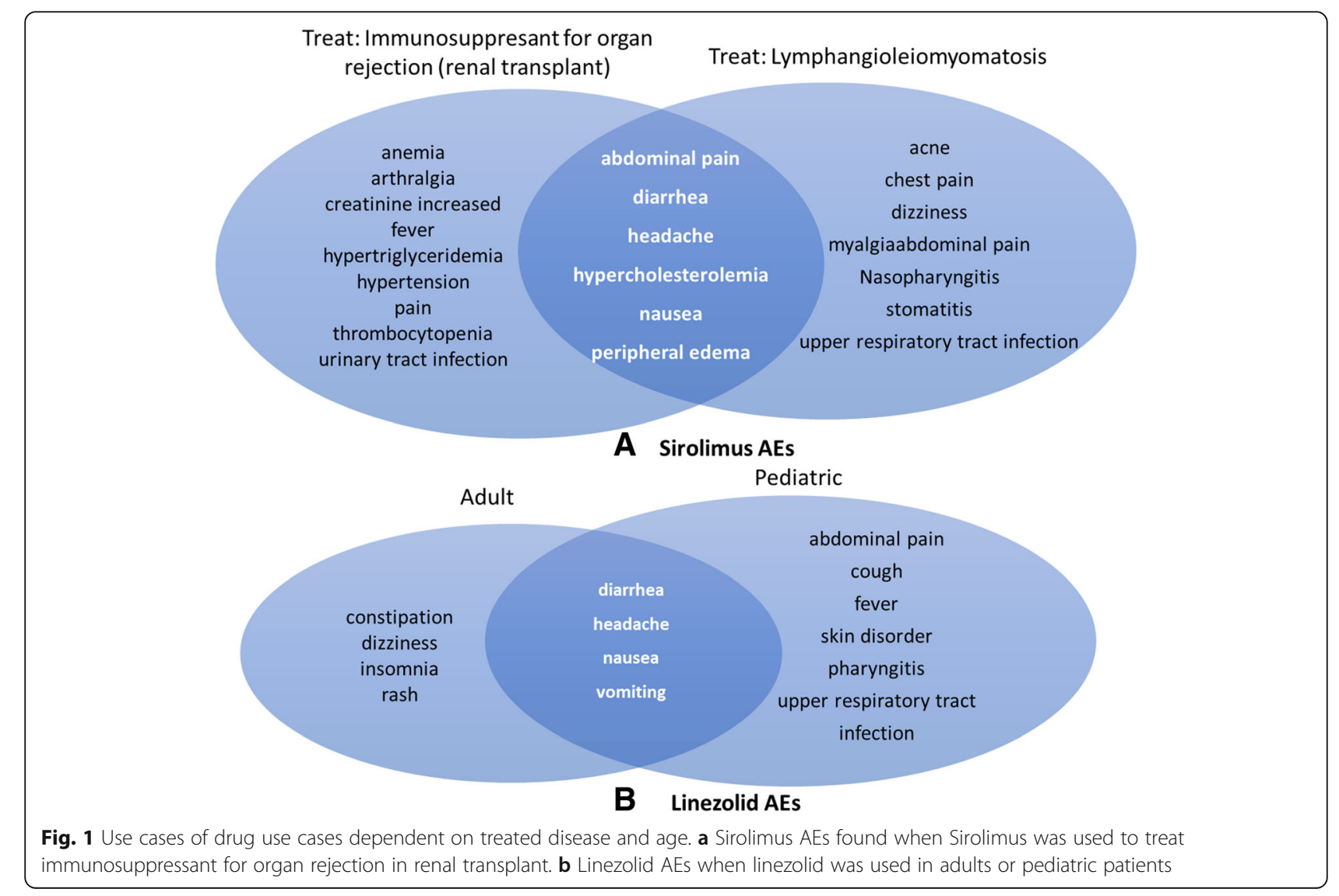




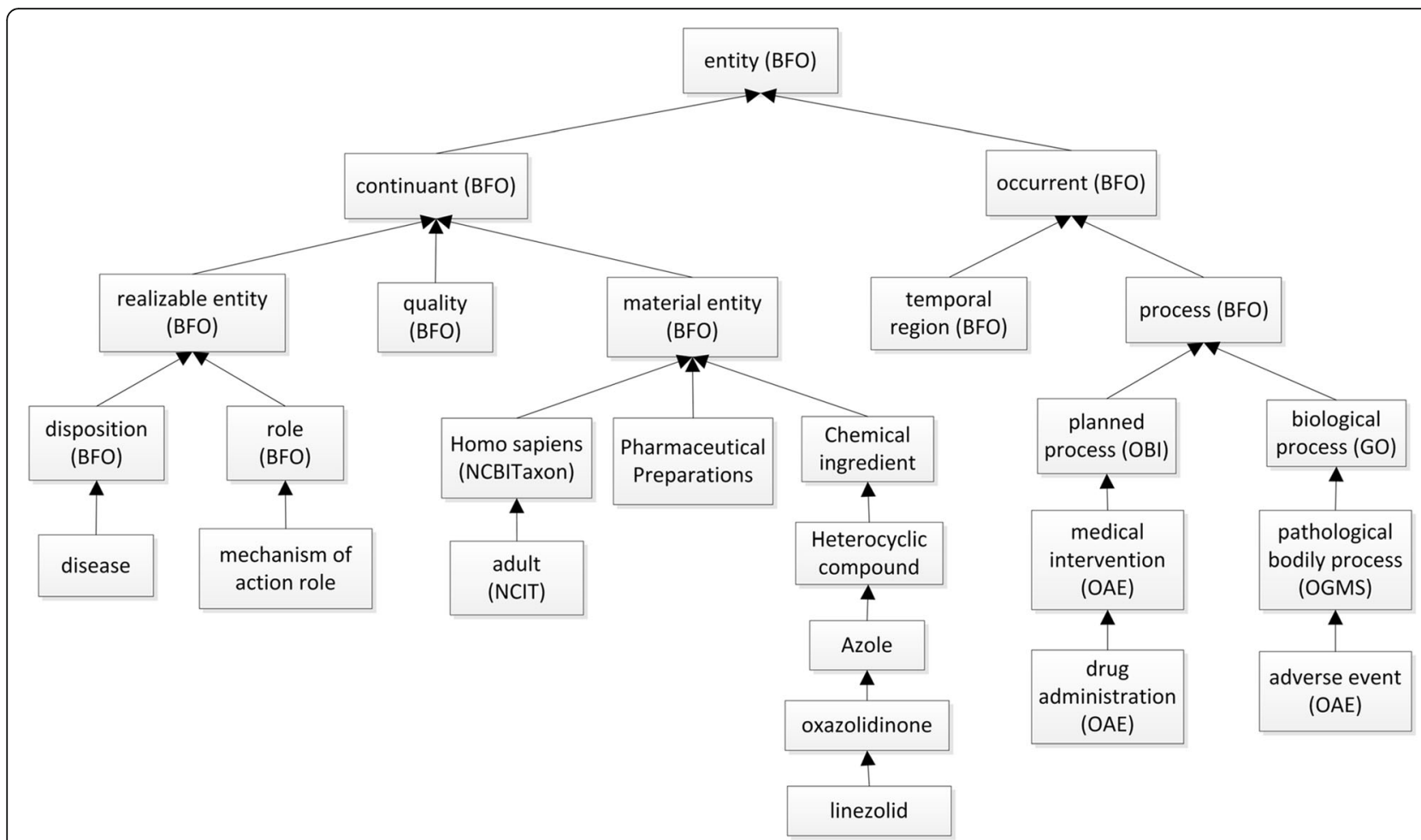

Fig. 2 ODAE top level hierarchy with exampled specific terms. Each arrow represents an "is a" relation. Those terms with no ontology indicated are terms from NDF-RT

ontology. BFO has two branches: continuant and occurrent. The BFO continuant represents time-independent entities such as material entity (e.g., drug), quality and roles. The BFO occurrent represents time-related entities such as process (e.g., adverse event and drug administration) and time. BFO is adopted by over 100 biomedical ontologies (http://basic-formal-ontology.org/ users.html). The usage of BFO makes ODAE easily and effectively integrated with other ontologies. ODAE also reuses terms from many ontologies. For example, ODAE reuses OAE to represent various AEs, the NCBI taxonomy ontology (NCBITaxon) [9] to represent human (i.e., Homo sapiens), and NDF-RT to represent drugs, drug ingredients, and mechanisms of action.

It is noted that the Disease Ontology (DOID) [43] and the Chemical Entities of Biological Interest (ChEBI) [44] have been recommended as the standard ontology to represent human diseases and chemicals, respectively. However, NDF-RT includes many diseases and the relations between drugs and diseases that may be treated by the drugs. Since such disease-drug information is not listed in DOID, we have also automatically extracted the disease-drug information from NDF-RT and use it in ODAE. Similarly, NDF-RT includes the terms of chemical ingredients as active components of drugs, which is not provided in ChEBI. Therefore, we also extracted the information of chemical ingredients from NDF-RT to ODAE. Our manual evaluation suggests that NDF-RT disease and chemical representations are also accurate and present the reality of the fields.

Figure 3a provides a general design pattern that links drug, drug chemical ingredient, mechanism of action, human group (e.g. adult) based on age, disease treated by drug, and AE. In RDF and OWL, a property is a binary relation. In our ODAE, we need to represent complex relations, called $n$-ary relations, among more than two entities. To represent such relations, we have designed and applied different object properties as indicated in the arrows in Fig. 3. The object properties 'used to treat disease (in adult)' and 'drug associated with $\mathrm{AE}$ (in adult)' are the shortcut relations that semantically and directly link drug to disease and $\mathrm{AE}$ in patient (e.g., adult patient), respectively. As an example, Fig. 3b uses drug Linezolid to illustrate how the general design pattern works out. Based on the general design pattern (Fig. 3a), we can generate axioms for Linezolid as follows:

LINEZOLID: ('drug associated with AE' some ('rash AE' and ('occurs in' some Adult and ('has disease' some 'Pneumonia, Bacterial'))))

The same information can also be presented using a simplified version for more efficient SPARQL query: 


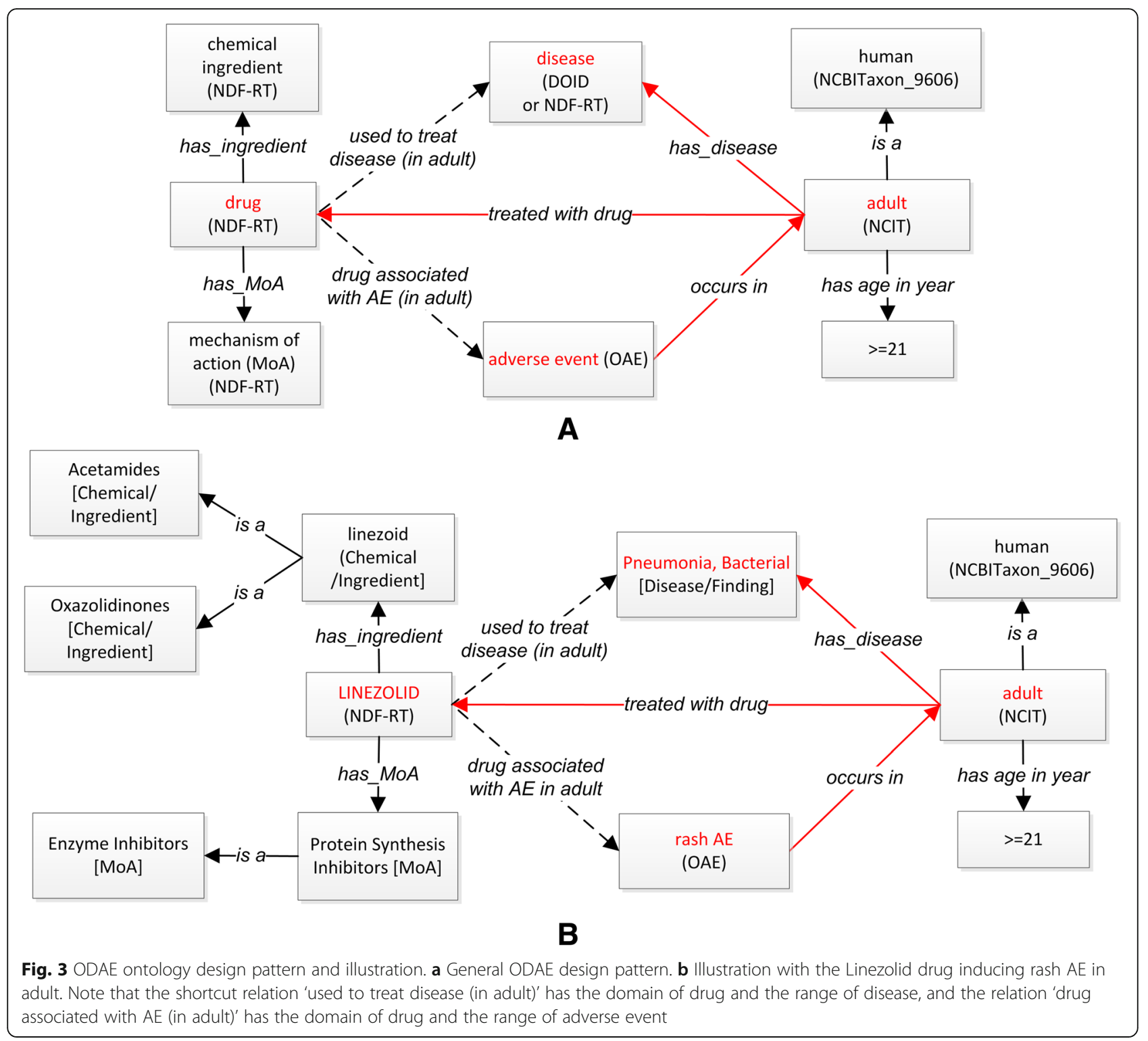

LINEZOLID: ('drug associated with $\mathrm{AE}$ in adult' some 'rash $\mathrm{AE}$ ') and ('used to treat disease' some 'Pneumonia, Bacterial')

Figure 4 further provides an ontology representation example using the drug Alosetron, which illustrates the ODAE hierarchy and the usage of design pattern (Fig. 3). Alosetron is a 5-HT3 antagonist used to treat diarrhea and abdominal discomfort that occurs in some women with irritable bowel syndrome [45]. As shown in Fig. 3, we can represent the same ADE information from the drug side or from the AE side. Figure 4a demonstrates how we can represent the ADE from the drug side, similar to the Linezolid example described above. To help users' browsing and analysis from different angles, Fig. $4 \mathrm{~b}$ illustrates the representation of the same ADE information from the AE side. From the ADE side, we can generate the following axiom:

'constipation AE': 'occurs in' some (Adult and (('has disease' some 'irritable bowel syndrome) and ('treated with drug' some ALOSETRON)))

Like ODNAE [29], ODAE represents the knowledge of drug adverse events from the FDA drug package insert documents. The detailed sample size information is typically available in package insert documents but not represented in ODAE. The knowledge represented in ODAE includes the statistically significant results out of the randomized controlled clinical trials described in the package insert documents. To provide the source of evidence and ensure the accuracy, 


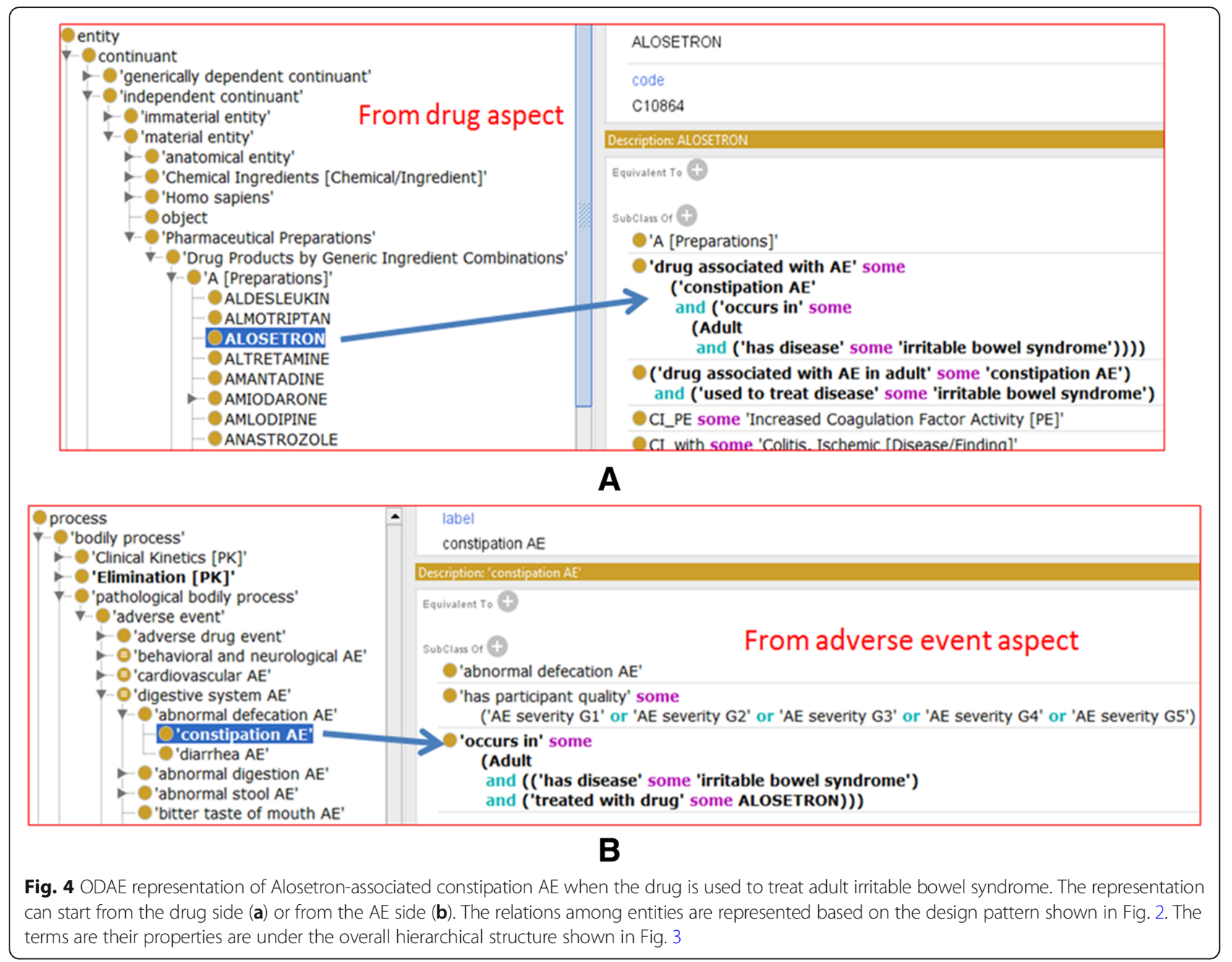

ODAE also includes the drug package insert documents as reference annotations.

\section{ODAE statistics and access}

As of January 12, 2019, ODAE has 3938 terms, including 3673 classes, 130 object properties, 18 data type properties, 114 annotation properties, and 3 instances. Most of the terms in ODAE are extracted and reused from over 20 ontologies (e.g., BFO, OAE, NCBITaxon, and DOID). ODAE only generates 15 new terms whose ontology identifiers having the "ODAE_" prefix. These 15 ODAE terms include 13 object properties, such as 'drug AE occurs in,' 'drug associated with AE', and 'treated with drug'. More ODAE statistics details can be found on the Ontobee website: http://www.ontobee.org/ontostat/ODAE.

It is noted that although ODAE does not have many new ODAE-specific terms, ODAE has generated many new semantical relations that link different drugs, AEs, human group with different age ranges (e.g., adult), and diseases to be treated by drugs (Figs. 3 and 4). In addition, ODAE semantically lists and annotates all these terms in an integrative hierarchical framework (Fig. 2).

ODAE is open-sourced and freely available. The ODAE ontology has been submitted to the Ontobee ontology repository and is openly available for visualization, browsing, and query on Ontobee: http:// www.ontobee.org/ontology/ODAE. The following website provides one example of the Ontobee display of the drug ALOSTERON's information in ODAE:

http://www.ontobee.org/ontology/ODAE?iri=http://evs. nci.nih.gov/ftp1/NDF-RT/NDF-RT.

owl\%23N0000148640. The information shown in Fig. 3a can also be seen on this Ontobee page, and more information is provided as well.

ODAE has also been deposited in the BioPortal website for web browsing and query: http://bioportal.

bioontology.org/ontologies/ODAE. 


\section{SPARQL query of the ODAE knowledge base}

The ODAE knowledge and its semantic links to other ontologies can be efficiently queried using SPARQL. Figure 5 provides an Ontobee SPARQL query example, which queries all the drugs in ODAE that function by interacting with ion channels, drug-treated diseases, and AEs when the drugs are used to treat the diseases in adult patients. The short SPARQL identified 8 neuropathy-inducing drugs that have the mechanisms of actions (MoAs) of 7 different ion channel interactions, and $36 \mathrm{AEs}$ are associated with these drugs when the drugs are used to treat 8 diseases in adults. The complete SPARQL results are available in the Additional File 1. One example is Phenytoin, an anti-seizure medication that functions by causing voltage-dependent block of voltage gated sodium channels [46]. EAST syndrome is a syndrome of seizures, sensorineural deafness, ataxia, mental retardation, and electrolyte imbalances [47]. When the drug is used to treat adult EAST syndrome patient, it can cause 7 AEs such as nystagmus, rash, somnolence, and speech disorder (Fig. 5). Another drug example is Diltiazem, a nondihydropyridine calcium channel blocker used in the treatment of hypertension, angina pectoris, and arrhythmia [48]. Our query found that Diltiazem is associated with asthenia $\mathrm{AE}$ when it is used to treat intermediate coronary syndrome in adults, and it is associated with 5 other AEs (i.e., pharyngitis, rhinitis, headache, constipation, and cough increased) when used to treat hypertension in adult patients. This example also shows that the same drug can induce different AEs when it is used to treat different diseases.

The above query example semantically links drugs, drug MoAs, AEs, and adult diseases treated by the drugs. Here the drugs and drug MoAs are recorded in NDF-RT, AEs represented in OAE, and diseases represented in DOID. Object properties are used to semantically link these terms to form specific knowledge information. Note that these drugs, MoAs, AEs, and diseases are organized in their own hierarchies. Based on the hierarchy, we can then query all possible ion channel interaction MOAs (e.g., sodium channel blocker) using

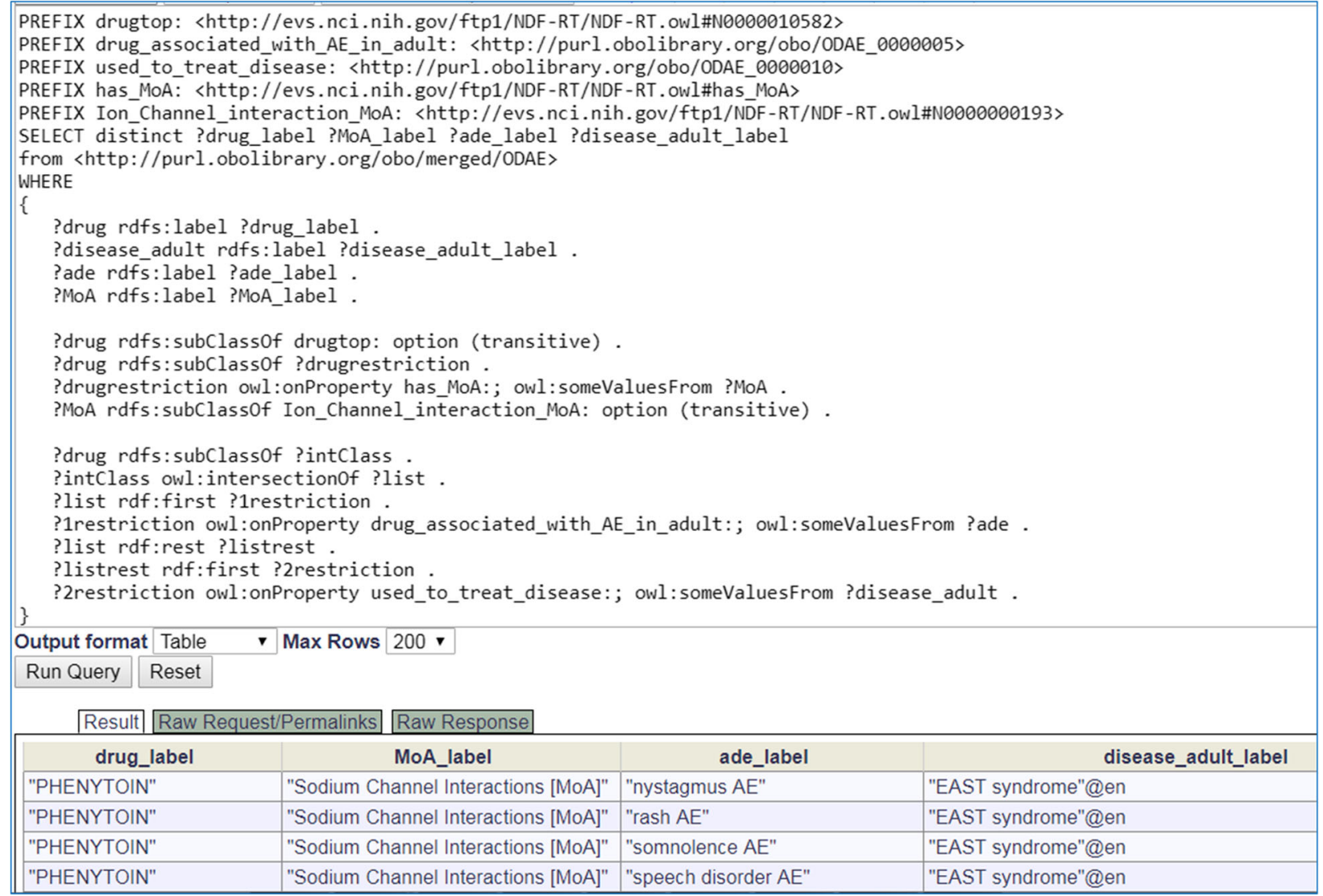

\begin{tabular}{||l|l|l|l|}
\hline \multicolumn{1}{|c|}{ drug_label } & \multicolumn{1}{c|}{ MoA_label } & \multicolumn{1}{c|}{ disease_adult_label } \\
\hline "PHENYTOIN" & "Sodium Channel Interactions [MoA]" & "nystagmus AE" & "EAST syndrome"@en \\
\hline "PHENYTOIN" & "Sodium Channel Interactions [MoA]" & "rash AE" & "EAST syndrome"@en \\
\hline "PHENYTOIN" & "Sodium Channel Interactions [MoA]" & "somnolence AE" & "EAST syndrome"@en \\
\hline "PHENYTOIN" & "Sodium Channel Interactions [MoA]" & "speech disorder AE" & "EAST syndrome"@en \\
\hline
\end{tabular}

Fig. 5 Example SPARQL query of ODAE. This query searches ODAE for all possible drugs that have some mechanism of action (MoA) related to ion channel interactions, and when used to treat some disease in adult patients, induce specific adverse events (AEs). Five related terms are defined using PREFIX. This query identified 8 drugs with 7 ion channel interaction MOAs, and these drugs are associated with 36 AEs when used to treat 8 diseases. Only part of results is shown in the screenshot. This query was performed using Ontobee SPARQL website (http://www.ontobee.org/sparql) 
the transitive query option (Fig. 5). Although not shown, more hierarchies (e.g., drug classification) can also be queried. Therefore, ODAE provides a rich semantic knowledge environment useful for flexible queries.

Furthermore, the SPARQL queried ODAE results can be used for statistical analysis. For example, we have recently generated a new ontology-based method to identify AE-specific drug class effect [27]. Such AE-specific drug class effect is defined to exist when all the drugs in a drug class (e.g., a specific MoA) are associated with an $\mathrm{AE}$, which can be formulated as a proportional class level ratio ("PCR") $=1$. The PCR score is the ratio between the number of drugs in a class that are associated with an $\mathrm{AE}$ and the total number of drugs in the class [27]. Based on the PCR method [27], we identified many AE-specific drug class effects from ODAE. For example, we found that the tyrosine kinase inhibitor MoA class has a class effect with several AEs, including rash, fever, nausea, edema, dyspnea, and vomiting AEs. All the three drugs that function with their MoAs under the MoA class, including IMATINIB (has_MoA Bcr-Abl Tyrosine Kinase Inhibitors [MoA]), ERLOTINIB (has_MoA HER1 Antagonists [MoA]), and TRASTUZUMAB (has_MoA HER2/Neu/cerbB2 Antagonists [MoA]), are associated with these AEs in drug-treated adult patients. We also found that 9 out of 10 drugs with the Immunologic and Biological Factors [MoA] are associated with the nausea AEs, leading to a PCR of 0.9 between the MoA class and the nausea AE. Such classification of AE-specific drug class effects rely on the semantic relations and hierarchical definitions in ODAE, demonstrating another usage of the ODAE knowledge in identifying possible causal associations.

\section{Discussion}

The contribution of this manuscript is multifold. First of all, we found and used an ontology-based approach to annotate all cases of neuropathy-inducing drug AEs given patient age and diseases, which are reported in the formal FDA-released drug package insert documents. Our study found that given different patient age and diseases, drugs tend to generate overlapping but different profiles of adverse events. Such a phenomenon may have important implication for how to report ADEs in public media (e.g., web reports). Second, we developed an Ontology of Drug Adverse Events (ODAE). ODAE includes an interoperable and integrative hierarchical structure and an ontology design pattern that logically links different types of entities, which were then applied to represent all aforementioned ADEs including those ADEs conditional to the changes of patient age and diseases to be treated. Third, our use case testing and ODAE evaluation found that ODAE can serve as a knowledge base of ADEs and be used for computer-assisted advanced queries. Since our ODAE approach is generic and scalable, it is also possible to apply the ODAE platform to represent other types of ADEs.

ODAE overlaps with but significantly differs from the ODNAE ontology. The Ontology of Drug Neuropathy Adverse events (ODNAE) focuses on ontological representation of drug-associated neuropathy AEs [29]. At current stage, ODAE and ODNAE include similar number of drugs. However, the ADEs represented in ODNAE are only neuropathy $\mathrm{AE}$ or its subclasses. Since neuropathy-inducing drugs also induce non-neuropathy AEs, ODNAE has its shortcoming in systematic representation of all possible AEs significantly associated with these over 200 drugs. ODAE first solved this issue. Since our recent analysis identified many ADEs that occur in a way dependent on patient age and diseases, ODAE has a new goal to logically and efficiently representing ADEs given patient age and disease, which is not done in ODNAE. To be more general and robust, our new ODAE design pattern is much simpler than the ODNAE pattern. For example, ODNAE includes many intermediate terms like 'bupropion-associated neuropathy $\mathrm{AE}$ ' (ODNAE_0000043). Using such logic, to deal with age and drug-treated disease-specific ADEs, ODAE would need to generate many new terms such as bupropion-associated neuropathy $\mathrm{AE}$ in adult (or other age range)' and 'bupropion-associated neuropathy $\mathrm{AE}$ when treating disease $\mathrm{X}$ ' However, such an approach would result in the generation of too many new terms in ODAE and is not scalable. Therefore, we decided not to include all these intermediate layer terms. Indeed, these intermediate terms were not used in any of the figures shown in the ODNAE paper [29]. Our evaluation found that the exclusion of these intermediate terms does not change the performance of robust queries of ODAE. Furthermore, while ODNAE focuses on neuropathy-inducing drugs, ODAE will become a general platform for representing AEs associated with other drugs in different domains.

Similarly, ODAE also differs from the Ontology of Cardiovascular Drug AEs (OCVDAE) [27]. OCVDAE targets on cardiovascular drugs used to treat cardiovascular diseases [27]. ODAE is more general. The design pattern in ODAE is also more robust than OCVDAE. One specific demonstration of OCVDAE is its application in analyzing drug class effect, which is defined exist when all the drugs in a drug class are associated with an AE. In the OCDAE paper, a mathematic proportional class level ratio (PCR) formula was developed and used to identify different types of drug class effect, such as the finding of cardiovascular drugs under the active transporter interactions class (including reserpine, indapamide, digoxin, and deslanoside) has statistically significant drug class effect on anorexia and diarrhea AEs [27]. At least theoretically, ODAE can also be used to analyze 
such drug class effects since ODAE also includes NDF-RT drug classifications and drug AEs. We will perform further studies to evaluate and possibly extent this potential.

ODAE is anticipated to play an important role in the future semantic ADE representation and drug safety studies. The Ontology of Adverse Events (OAE), developed by our group and collaborators around the world, has been a major contribution in recent years that lays out the semantic framework and representation of various adverse events. The OAE general framework has been applied to represent and study AEs associated with vaccines in the Ontology of Vaccine Adverse Events (OVAE) [28], neuropathy-inducing drugs in ODNAE [29], and drugs used to treat cardiovascular diseases in OCVDAE [27]. OAE also provides a way to link different domains of entities such as medical administration, anatomic entities, phenotypes, and patient qualities, and cross-reference other controlled terminologies such as the Common Terminology Criteria for Adverse Events (CTCAE) [49] and the Medical Dictionary for Regulatory Activities (MedDRA) [50]. It has become clear that biological ontologies support the integration of biological knowledge for learning and predicting ADEs [51]. ODAE provides a new and integrative ontology towards systematical representation of all ADEs and how these ADEs are related to drugs, drug mechanisms of action, drug chemical ingredients, etc. ODAE can thus serve as an ADE knowledge base and a hub to link other related fields of knowledge and studies.

A future direction is how to further study and represent the molecular mechanisms of the adverse drug events. Our Fig. 5 SPARQL query example demonstrates how we can query ODAE for the semantic relations among drugs, drug MoAs, and diseases treated by the drugs, and AEs that occurs in adults after treatment with the drugs. The ontology hierarchy provides us a feasible strategy to examine the effect of a specific group of entities (e.g., the group of ion channel interaction MoAs). Similarly, we can also search for information about other related entities such as drug ingredients and their hierarchies. It is noted that the mechanisms of why age and existing disease affect the ADEs are often unclear and require extensive studies. Humans with different age stages have many different physiological characteristics, habits, and living environments. The existence of diseases in patients may also change the preference and status of immune systems and metabolisms, which may also affect the body reactions to the administration of a drug. More knowledge obtained from experimental studies can be added to ODAE in the future. We have also shown in the Results section how to analyze AE-specific drug class effects [27] using ODAE, which can suggest causal association between the $\mathrm{AE}$ and the drug classification (e.g., MoA). Therefore, ODAE provides a knowledge base and platform to represent, integrate, and analyze different drugs and drug ingredients, diseases, AEs, mechanisms, and their intricate relations among these entities.

\section{Conclusion}

In this study, we first collected different AEs associated with 224 neuropathy-inducing drugs by manual annotation of FDA-released drug package insert documents. Our study identified many ADEs conditional to the patient age and diseases to be treated with drugs. We then developed an ontological design pattern for representation of the relations between drugs, ages, diseases to be treated, and AEs, and based on develop the Ontology of Drug Adverse Events (ODAE). ODAE reuses existing ontologies and logically represents all the 224 drugs, drug chemical ingredients and their features, and their associated AEs. ODAE can be used for visualization and queries of ADEs. Overall, ODAE is a novel ontology and can serve as a new platform for representing the AEs and related information for other drugs.

\section{Additional file}

Additional file 1: Complete results of SPARQL query in Fig. 5.

(XLSX $17 \mathrm{~kb})$

\section{Abbreviations}

AE: Adverse event; BFO: Basic Formal Ontology; FDA: Food and Drug Administration; NCBITaxon: NCBI taxonomy ontology; OAE: Ontology of Adverse Events; OBO: Open Biological/Biomedical Ontologies; OCVDAE: Ontology of Cardiovascular Drug AEs; ODAE: Ontology of Drug Adverse Events; ODNAE: Ontology of Drug Neuropathy Adverse events; OVAE: Ontology of Vaccine Adverse Events; OWL2: Web Ontology Language; RDF: Resource Description Framework; SPARQL: SPARQL Protocol and RDF Query Language

\section{Acknowledgements}

Not applicable.

\section{Funding}

This project was partly supported by NIH-NIAID grant (1R01AI081062), Guiyang science and technology programs ZKHT [20151001] S78 and ZKHT [20161001] 09, Guizhou science and technology programs QKH SY [2010] 3044, and the University of Michigan Undergraduate Research Opportunity Program (UROP). The Guizhou Provincial People's Hospital provided financial support for HY's half-a-year research visit in the University of Michigan. Publication costs are funded by Guiyang science and technology program ZKHT [20151001] S78.

\section{Availability of data and materials}

The ODAE source in available in the ODAE project website at GitHub: https:// github.com/ODAE-ontology/ODAE.

\section{About this supplement}

This article has been published as part of BMC Bioinformatics Volume 20 Supplement 7, 2019: Selected papers from the 12th International Conference on Computational Systems Biology (ISB 2018). The full contents of the supplement are available online at https://bmcbioinformatics.biomedcentral. com/articles/supplements/volume-20-supplement-7. 


\section{Authors' contributions}

HY: Domain expert of respiratory diseases and ADEs, ODAE developer, use case development and evaluation, result interpretation SN and NG: Data collection, manual annotation, ODAE development, and data analysis EO: ODAE ontology development, evaluation, and analysis XY and XZ: Domain expert of respiratory diseases and ADEs YH: Project design, primary ODAE developer, use case testing, and data interpretation. All authors participated in discussion, manuscript preparation and editing, and approved the manuscript.

\section{Ethics approval and consent to participate}

Not applicable.

\section{Consent for publication}

Not applicable.

\section{Competing interests}

The authors declare that they have no competing interests.

\section{Publisher's Note}

Springer Nature remains neutral with regard to jurisdictional claims in published maps and institutional affiliations.

\section{Author details}

'Department of Pulmonary and Critical Care Medicine, Guizhou Provincial People's Hospital, Guiyang 550002, Guizhou, China. ${ }^{2}$ Guizhou University Medical College, Guiyang 550025, Guizhou, China. ${ }^{3}$ College of Literature, Science, and the Arts, University of Michigan, Ann Arbor, Ml 48109, USA. ${ }^{4}$ College of Pharmacy, University of Michigan, Ann Arbor, MI 48109, USA. ${ }^{5}$ Department of Computational Medicine and Bioinformatics, University of Michigan Medical School, Ann Arbor, Ml 48109, USA. ${ }^{6}$ Unit for Laboratory Animal Medicine, Department of Microbiology and Immunology, Center for Computational Medicine and Bioinformatics, and Comprehensive Cancer Center, University of Michigan Medical School, Ann Arbor, Ml 48109, USA

Published: 1 May 2019

\section{References}

1. Bonn D. Adverse drug reactions remain a major cause of death. Lancet. 1998;351(9110):1183. https://doi.org/10.1016/50140-6736(98)23016-9.

2. Bouvy JC, De Bruin ML, Koopmanschap MA. Epidemiology of adverse drug reactions in Europe: a review of recent observational studies. Drug Saf. 2015; 38(5):437-53. https://doi.org/10.1007/s40264-015-0281-0.

3. Shepherd G, Mohorn P, Yacoub K, May DW. Adverse drug reaction deaths reported in United States vital statistics, 1999-2006. Ann Pharmacother. 2012:46(2):169-75. https://doi.org/10.1345/aph.1P592.

4. Blake JA, Bult CJ. Beyond the data deluge: data integration and bioontologies. J Biomed Inform. 2006;39(3):314-20. https://doi.org/10.1016/jj.jbi. 2006.01.003.

5. Hoehndorf R, Schofield PN, Gkoutos GV. The role of ontologies in biological and biomedical research: a functional perspective. Brief Bioinform. 2015. https://doi.org/10.1093/bib/bbv011.

6. Bodenreider O. Biomedical ontologies in action: role in knowledge management, data integration and decision support. Yearb Med Inform. 2008; 17:67-79

7. Schulz S, Balkanyi L, Cornet R, Bodenreider O. From concept representations to ontologies: a paradigm shift in health informatics? Healthcare Inform Res. 2013;19(4):235-42. https://doi.org/10.4258/hir.2013.19.4.235.

8. Xiang Z, Courtot M, Brinkman RR, Ruttenberg A, He Y. OntoFox: web-based support for ontology reuse. BMC Res Notes 2010;3:175:1-12. doi:1756-05003-175 [pii] https://doi.org/10.1186/1756-0500-3-175.

9. NCBITaxon: An ontology representation of the NCBI organismal taxonomy. http://obofoundry.org/ontology/ncbitaxon.html, Accessed 23 Feb 2019.

10. Demir E, Cary MP, Paley S, Fukuda K, Lemer C, Vastrik I, et al. The BioPAX community standard for pathway data sharing. Nat Biotechnol. 2010;28(9): 935-42. https://doi.org/10.1038/nbt.1666.

11. Ashburner M, Ball CA, Blake JA, Botstein D, Butler H, Cherry JM, et al. Gene ontology: tool for the unification of biology. The Gene Ontology Consortium. Nat Genet. 2000;25(1):25-9.
12. Bandrowski A, Brinkman R, Brochhausen M, Brush MH, Bug B, Chibucos MC, et al. The Ontology for Biomedical Investigations. PLoS ONE. 11(4): e0154556. https://doi.org/10.1371/journal.pone.0154556

13. Zheng J, Manduchi E, Stoeckert CJ Jr. Development of an Application Ontology for Beta Cell Genomics based On the Ontology for Biomedical Investigations. The 4th International Conference on Biomedical Ontology (ICBO-2013). 2013;1060:62-7.

14. Brinkman RR, Courtot M, Derom D, Fostel J, He Y, Lord P, et al. Modeling biomedical experimental processes with OBI. J Biomed Semantics. 2010; 1(Suppl 1):S7. https://doi.org/10.1186/2041-1480-1-S1-S7.

15. Dugan VG, Emrich SJ, Giraldo-Calderon Gl, Harb OS, Newman RM, Pickett $\mathrm{BE}$, et al. Standardized metadata for human pathogen/vector genomic sequences. PLoS One. 2014;9(6):e99979. https://doi.org/10.1371/ journal.pone.0099979.

16. Sarntivijai S, Lin Y, Xiang Z, Meehan TF, Diehl AD, Vempati UD, et al. CLO: The Cell Line Ontology. J Biomed Semantics. 2014;5:37. https://doi.org/10. 1186/2041-1480-5-37.

17. Gonzalez-Beltran A, Maguire E, Sansone SA, Rocca-Serra P. linkedISA: semantic representation of ISA-Tab experimental metadata. BMC Bioinformatics. 2014; 15(Suppl 14):S4. https://doi.org/10.1186/1471-2105-15-S14-S4.

18. Malladi VS, Erickson DT, Podduturi NR, Rowe LD, Chan ET, Davidson JM, et al. Ontology application and use at the ENCODE DCC. Database. 2015;2015. https://doi.org/10.1093/database/bav010.

19. Stodden V, Guo P, Ma Z. Toward reproducible computational research: an empirical analysis of data and code policy adoption by journals. PLoS One. 2013;8(6):e67111. https://doi.org/10.1371/journal.pone.0067111.

20. Wilkinson MD, Dumontier M, Aalbersberg IJ, Appleton G, Axton M, Baak A, et al. The FAIR guiding principles for scientific data management and stewardship. Scientific data. 2016;3:160018. https://doi.org/10.1038/sdata.2016.18.

21. He Y, Xiang Z, Zheng J, Lin Y, Overton JA, Ong E. The eXtensible ontology development (XOD) principles and tool implementation to support ontology interoperability. J Biomed Semantics. 2018;9(1):3. https://doi.org/ 10.1186/s13326-017-0169-2.

22. He Y, Xiang Z, Sarntivijai S, Toldo L, Ceusters W. AEO: a realism-based biomedical ontology for the representation of adverse events. Adverse Event Representation Workshop, International Conference on Biomedical Ontologies (ICBO-2011); 2011 July 26-30. Buffalo, NY, USA: CEUR Workshop Proceedings; 2011.

23. Sarntivijai S, Xiang Z, Shedden KA, Markel H, Omenn GS, Athey BD, et al. Ontology-based combinatorial comparative analysis of adverse events associated with killed and live influenza vaccines. PLoS One. 2012;7(11): e49941. https://doi.org/10.1371/journal.pone.0049941.

24. Hastings J, Jeliazkova N, Owen G, Tsiliki G, Munteanu CR, Steinbeck C, et al. eNanoMapper: harnessing ontologies to enable data integration for nanomaterial risk assessment. J Biomed Semantics. 2015;6:10. https://doi. org/10.1186/s13326-015-0005-5.

25. Tao C, He Y, Yang H, Poland GA, Chute CG. Ontology-based time information representation of vaccine adverse events in VAERS for temporal analysis. J Biomed Semantics. 2012;3(1):13. https://doi.org/10. 1186/2041-1480-3-13.

26. Gurulingappa H, Mateen-Rajput A, Toldo L. Extraction of potential adverse drug events from medical case reports. J Biomed Semantics. 2012;3(1):15. https://doi.org/10.1186/2041-1480-3-15.

27. Wang L, Li M, Xie J, Cao Y, Liu H, He Y. Ontology-based systematical representation and drug class effect analysis of package insert-reported adverse events associated with cardiovascular drugs used in China. Sci Rep. 2017;7(1):13819. https://doi.org/10.1038/s41598-017-12580-4.

28. Marcos E, Zhao B, He Y. The ontology of vaccine adverse events (OVAE) and its usage in representing and analyzing adverse events associated with USlicensed human vaccines. J Biomed Semantics. 2013;4(1):40. https://doi.org/ 10.1186/2041-1480-4-40

29. Guo A, Racz R, Hur J, Lin Y, Xiang Z, Zhao L, et al. Ontology-based collection, representation and analysis of drug-associated neuropathy adverse events. Journal of biomedical semantics. 2016;7:29. https://doi.org/ 10.1186/s13326-016-0069-x.

30. Schriml LM, Arze C, Nadendla S, Chang YW, Mazaitis M, Felix V, et al. Disease ontology: a backbone for disease semantic integration. Nucleic Acids Res. 2012;40(Database issue):D940-6. https://doi.org/10.1093/nar/gkr972.

31. Peters $L B, B a h r N$, Bodenreider $O$. Evaluating drug-drug interaction information in NDF-RT and DrugBank. J Biomed Semantics. 2015;6:19. https://doi.org/10.1186/s13326-015-0018-0. 
32. Smith B, Ashburner M, Rosse C, Bard J, Bug W, Ceusters W, et al. The OBO foundry: coordinated evolution of ontologies to support biomedical data integration. Nat Biotechnol. 2007;25(11):1251-5. https:/doi.org/10.1038/nbt1346.

33. Mungall CJ, Torniai C, Gkoutos GV, Lewis SE, Haendel MA. Uberon, an integrative multi-species anatomy ontology. Genome Biol. 2012;13(1):R5. https://doi.org/10.1186/gb-2012-13-1-r5.

34. He Y, Sarntivijai S, Lin Y, Xiang Z, Guo A, Zhang S, et al. OAE: the ontology of adverse events. J Biomed Semantics. 2014;5:29. https://doi.org/10.1186/ 2041-1480-5-29.

35. Xiang Z, Zheng J, Lin Y, He Y. Ontorat: Automatic generation of new ontology terms, an-notations, and axioms based on ontology design patterns. J Biomed Semantics. 2015;6(1):4 (10 pages). https://doi.org/10. 1186/2041-1480-6-4

36. Ong E, Xiang Z, Zhao B, Liu Y, Lin Y, Zheng J, et al. Ontobee: a linked ontology data server to support ontology term dereferencing, linkage, query and integration. Nucleic Acids Res. 2017;45(D1):D347-D52. https://doi. org/10.1093/nar/gkw918.

37. W3C. OWL 2 Web Ontology Language Quick Reference Guide (Second Edition), W3C Recommendation 11 December 2012. 2012: http://www.w3. org/TR/owl2-quick-reference/. Accessed 10 Aug 2018.

38. Harris S, Seaborne A. SPARQL 1.1 Query Language, W3C Recommendation 21 March 2013. 2013: URL: http://www.w3.org/TR/sparql11-query/, Accessed 21 Oct 2017.

39. Halleck F, Duerr M, Waiser J, Huber L, Matz M, Brakemeier S, et al. An evaluation of sirolimus in renal transplantation. Expert Opin Drug Metab Toxicol. 2012:8(10):1337-56. https://doi.org/10.1517/17425255.2012.719874.

40. Peng ZF, Yang L, Wang TT, Han P, Liu ZH, Wei Q. Efficacy and safety of sirolimus for renal angiomyolipoma in patients with tuberous sclerosis complex or sporadic lymphangioleiomyomatosis: a systematic review. J Urol. 2014;192(5):1424-30. https://doi.org/10.1016/j.juro.2014.04.096.

41. Zahedi Bialvaei A, Rahbar M, Yousefi M, Asgharzadeh M, Samadi KH. Linezolid: a promising option in the treatment of gram-positives. J Antimicrob Chemother. 2017;72(2):354-64. https://doi.org/10.1093/jac/dkw450.

42. Grenon P. Spatio-temporality in Basic Formal Ontology. In: Grenon P, editor. IFOMIS reports. Leipzig: Institute for Formal Ontology and Medical Information Science at the Faculty of Medicine of the University of Leipzig; 2003. p. 89.

43. Kibbe WA, Arze C, Felix V, Mitraka E, Bolton E, Fu G, et al. Disease ontology 2015 update: an expanded and updated database of human diseases for linking biomedical knowledge through disease data. Nucleic Acids Res. 2015;43(Database issue):D1071-8. https://doi.org/10.1093/nar/gku1011.

44. Hastings J, de Matos P, Dekker A, Ennis M, Harsha B, Kale N, et al. The ChEBI reference database and ontology for biologically relevant chemistry: enhancements for 2013. Nucleic Acids Res. 2013:41(Database issue):D45663. https://doi.org/10.1093/nar/gks1146.

45. Bleser S. Alosetron for severe diarrhea-predominant irritable bowe syndrome: improving patient outcomes. Curr Med Res Opin. 2011;27(3):50312. https://doi.org/10.1185/03007995.2010.547933.

46. Rogawski MA, Loscher W. The neurobiology of antiepileptic drugs. Nat Rev Neurosci. 2004:5(7):553-64. https://doi.org/10.1038/nrn1430

47. Bockenhauer D, Feather S, Stanescu HC, Bandulik S, Zdebik AA, Reichold M, et al. Epilepsy, ataxia, sensorineural deafness, tubulopathy, and KCNJ10 mutations. N Engl J Med. 2009;360(19):1960-70. https://doi.org/10.1056/ NEJMoa0810276.

48. O'Connor SE, Grosset A, Janiak P. The pharmacological basis and pathophysiological significance of the heart rate-lowering property of diltiazem. Fundam Clin Pharmacol. 1999;13(2):145-53.

49. Wong MU, Racz R, Ong E, He Y. Towards precision informatics of pharmacovigilance: OAE-CTCAE mapping and OAE-based representation and analysis of adverse events in patients treated with cancer drugs. AMIA Annual Symposium proceedings / AMIA Symposium AMIA Symposium. 2017;2017:1793-801.

50. Sarntivijai S, Zhang S, Jagannathan DG, Zaman S, Burkhart KK, Omenn GS, et al. Linking MedDRA-coded clinical phenotypes to biological mechanisms by the ontology of adverse events: a pilot study on tyrosine kinase inhibitors. Drug Saf. 2016. https://doi.org/10.1007/s40264-016-0414-0.

51. Zaman S, Sarntivijai S, Abernethy DR. Use of biomedical ontologies for integration of biological knowledge for learning and prediction of adverse drug reactions. Gene regulation and systems biology. 2017;11: 1177625017696075. https://doi.org/10.1177/1177625017696075.

\section{Ready to submit your research? Choose BMC and benefit from:}

- fast, convenient online submission

- thorough peer review by experienced researchers in your field

- rapid publication on acceptance

- support for research data, including large and complex data types

- gold Open Access which fosters wider collaboration and increased citations

- maximum visibility for your research: over $100 \mathrm{M}$ website views per year

At $\mathrm{BMC}$, research is always in progress.

Learn more biomedcentral.com/submissions 